\section{Quantum materials: Where many paths meet}

\author{
By Philip Ball
}

$A^{11}$ matter, in the end, must be explained by quantum mechanics, which describes how atoms bind and electrons interact at a fundamental level. Typically, the quantum behavior can be approximated by a classical description, in which atoms become balls that stick together in welldefined arrangements via simple forces and vibrate much like balls on springs.

Sometimes, however, that is not so. In some materials, the quantum aspects assert themselves tenaciously, and the only way to fully understand how the material behaves is to keep the quantum in view. Such substances are now grouped together under the banner of quantum materials.

The US Department of Energy (DOE) describes quantum materials as "solids with exotic physical properties, arising from the quantum mechanical properties of their constituent electrons, that have great scientific and/or technological potential." It's a diverse class of materials - so much so that some question whether the designation is meaningful. It includes well-advertised materials such as superconductors and graphene, along with ones with less familiar names: topological insulators, Weyl semimetals, quantum spin liquids, and spin ices.

A collection of oddballs they may be, but quantum materials are both a treasure trove of interesting physics and a potential source of useful substances. And while the compositions and behaviors of quantum materials cover a wide spectrum, some common themes recur. Many of them derive their properties from reduced dimensionality, in particular from confinement of electrons to two-dimensional (2D) sheets. In many, magnetism and electronic structure interact in curious ways. In particular, they tend to be materials in which electrons cannot be considered as independent particles but act as collective states dubbed quasiparticles.
Aside from the matter of their intrinsic properties, however, what tends to unite quantum materials is who is interested in them. The community of researchers that 20 years ago was grappling with high-temperature superconductivity is likely today to be pondering topological insulators and Weyl semimetals. A somewhat distinct community that used to focus around the 1980s on the exotic 2D phenomenon called the quantum Hall effect is now finding common cause. And quantum materials draw inspiration from a third direction too, apparently unlikely at first flush: particle physics, within which some unusual types of fundamental particles proposed around the mid-to-late 20th century are now finding analogues in the quasiparticles of condensed matter.

This is not a matter of leaping onboard the latest fancy-titled bandwagon. Rather, it is a reflection of a common experience in physics, whereby concepts developed to explore one phenomenon turn out to be a subset of more general principles. Quantum materials reveal that properties once thought to be quirks confined to exotic conditions are in fact a significant feature of the materials universe. To turn those ideas into real materials, meanwhile, demands expertise from other fields, involving the skills of synthetic solid-state materials chemistry and crystal growth. This union of fundamental physics and practical materials science is turning into one of the most vibrant areas of physical science today. Most of all, it offers a playground for finding and exploring new physics. But there could be practical benefits too: new electronic devices, and, in particular, new possibilities for building quantum computers, which would exploit the rules of quantum mechanics to achieve computational power far in excess of anything the classical computers of today can attain.

\section{Early promise}

Arguably, it all began with superconductivity. It was recognized in the 1950 s that the ability of some materials to conduct electricity without resistance is a phenomenon that demands a quantum explanation. The theory put forward by John Bardeen, Leon Cooper, and Robert Schrieffer (BCS) in 1957 invoked an interaction between mobile conduction electrons, mediated by vibrations of the crystal lattice that unites them into pairs, called Cooper pairs. These pairs can be considered as quasiparticles, which - in contrast to electrons themselves-belong to the fundamental class of particles called bosons, which have integer values of quantum spin.

This makes all the difference. Particles with half-integer spin (fermions), like lone electrons, cannot occupy the same quantum state. However, bosons can, and so Cooper pairs can condense into a quantum state in which all the electrons can be described by a single quantum wave function. In effect, they become a collective entity, which is then immune to the disturbing effects of electron scattering from the crystal lattice - the source of energy dissipation and electrical resistance. But this collective state can only be sustained at low temperatures; too much thermal noise, and the Cooper pairs are broken, and the material becomes a regular metal.

Here, then, is a behavior dictated by quantum mechanical correlations between electrons: the trademark signature of many quantum materials. Yet BCS theory did not seem to work for the class of "high-temperature" superconductors discovered by Georg Bednorz and K. Alex Müller of IBM's Zurich research laboratories in 1986, some of which proved to have superconducting transition temperatures much higher than the boiling point of liquid nitrogen $(77 \mathrm{~K})$. These materials are not metals but ceramics: metal oxides with a characteristic layered structure, of which the paradigmatic example was lanthanum copper oxides.

Despite several decades of work, there is still no fully satisfactory consensus view of how high-temperature 
superconductors work. "The complexity of the materials has made arriving at simple models difficult," says physicist David Ceperley of the University of Illinois at Urbana-Champaign. All the same, most researchers agree that the electron correlations that must surely lie at the root of the phenomenon are related to both the layered, quasi-2D crystal structure of the materials and to their magnetic behavior.

Meanwhile, another strand of exotic quantum properties was evolving in a different arena. In 1980, Klaus von Klitzing showed that at low temperatures, the Hall effect-an effect known for a century, in which a voltage across an electrical conductor is created by the influence of a magnetic field on the electron pathscan become quantized. That's to say, the conductance of a thin, quasi-2D conductor may change in stepwise jumps as the strength of a transverse magnetic field is increased: the quantum Hall effect (QHE). Qualitatively, this quantization is the result of electrons in the 2D material being confined to discrete looping orbits, not unlike those postulated for simple theories of the quantum atom, but much larger in size. These electron states owe their stability to the topological properties of the electronic band structure in the material. They are in some ways analogous to the topological defects that may appear in crystal structures, such as screw dislocations, which, like the whorled crown of hairs on your head, are irreducible consequences of shape. These structures are said to be "topologically protected."

Von Klitzing won the 1985 Nobel Prize in Physics for his work (it went to Bednorz and Müller in 1987), and, subsequently, more exotic variants of the QHE were discovered. Robert B. Laughlin, Horst L. Störmer, and Daniel C. Tsui won the 1998 physics Nobel for showing that under certain conditions, electrons in a $2 \mathrm{D}$ material that are free to move more or less without impediment $-\mathrm{a} 2 \mathrm{D}$ electron gas can condense into quasiparticle states that also show the QHE, but act as if they are electrons with fractional electric charge. This behavior is called the fractional quantum Hall effect (FQHE). It's still not fully understood, but again collectivity and topology of the electron states hold the key.

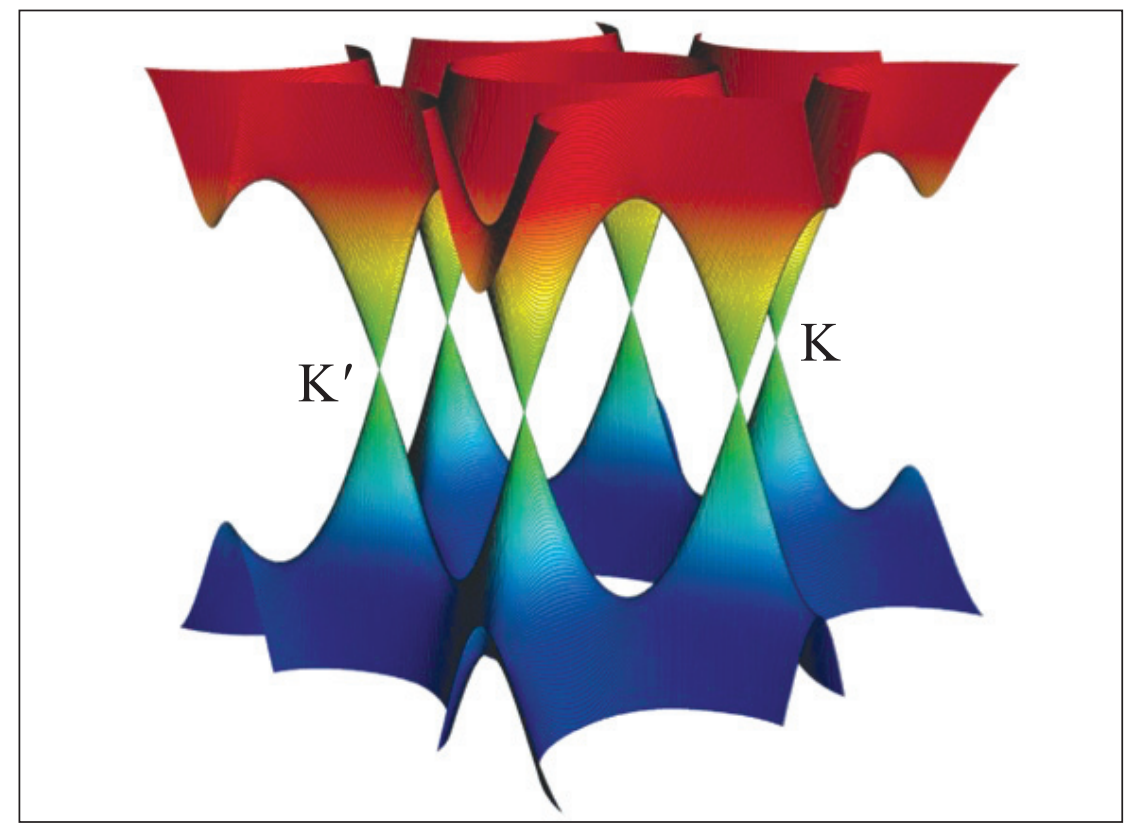

Figure 1. The band structure of graphene. The valence (blue/green) and conduction (red/yellow) bands touch at points $\mathrm{K}$ and $\mathrm{K}^{\prime}$ in momentum space.

\section{Kissing cones}

The connections between high-temperature superconductors, the QHE, and 2D electron gases are, in retrospect, clear enough: in particular, the reduced dimensionality, interaction of electronic and magnetic or electron-spin properties, and the importance of electron correlations and quasiparticle descriptions.

These aspects all come together in the much-vaunted "wonder material" graphene, which is also perhaps the most familiar and celebrated quantum material today. These 2D sheets of pure carbon, the atoms united into hexagonal rings long familiar from the mundane parent material graphite, have been proposedsome would say hyped - as a fabric for the next generation of electronic circuitry and touchscreens.

While the real value of graphene in that applied arena remains to be seen, few physicists would dispute that, as a playground for ideas in basic physics, graphene's potential is already proven. Indeed, it is really for elucidating the strange and often surprising electronic properties of this material - and not for their convenient way of making it by stripping individual layers from graphite using Scotch tape - that Andre Geim and
Konstantin Novoselov of The University of Manchester in the UK were awarded the 2010 Nobel Prize in Physics.

From a chemist's point of view, the extended network of conjugated bonds in the graphene sheet creates pathways for mobile electrons that give graphene its electrical conductivity. To the solid-state physicist, more used to describing electronic structure in terms of band theory, the picture is more complex. The peculiarity of graphene is that it has the fully filled valence band and empty conduction band of a semiconductor, but with a bandgap between these states that falls to zero at a particular value of electron momentum. That is the defining characteristic of a semimetal.

That graphene should be a gapless semiconductor has been recognized since its electronic band structure was first calculated in 1947—well before graphene was recognized as a distinct substance rather than just the hypothetical 2D "unit" of graphite. This band structure is typically described in terms of the so-called Fermi surface: loosely speaking, the surface of the volume that electrons in the material occupy in momentum space. For graphene, the Fermi surface sits right at the point 
where the conduction and valence bands touch like two cones "kissing" at their apex, and so in theory it seems to have zero area, making graphene a semiconductor (see Figure 1). In fact, some of the electron density can leak into the conduction band at these points due to quantum effects, making graphene electrically conductive even at zero temperature.

The shape of the Fermi surface means that the mobile electrons interact to form collective quasiparticles (see Table I) that act as though they have no mass. ${ }^{1}$ This enables them to achieve very high speeds - a significant fraction of the speed of light - giving graphene a large electron mobility that could be very useful for high-frequency electronic devices. The quasiparticles must be described by the relativistic form of quantum mechanics devised by physicist Paul Dirac in the late 1920s: They are known as Dirac fermions, and they were in fact one of the predictions of Dirac's theory. Graphene is thus called a 2D "Dirac semimetal."

This sort of relativistic behavior of particles is more familiar to particle physicists. Indeed, some quantum relativistic effects that are hard to achieve in particle physics turn out to be easier to arrange in graphene. One of them is called Klein tunneling, a variation on quantum tunneling of particles through barriers, in which, for massless relativistic particles, a barrier can become virtually transparent regardless of its height and thickness. It is because of this effect, which was observed in $2013,{ }^{2}$ that the charge carriers in the 2D structures can remain highly mobile even in the presence of potential scattering sites. On the other hand, this transparency of barriers is a problem for trying to make graphene transistors (at least from single-layer graphene), because it means that the gates that control charge-carrier motion in conventional transistors cannot be fully closed.

There is no reason why Dirac semimetals have to be $2 \mathrm{D}$, although the band structure and the Fermi surface become rather tricky to picture in three dimensions. In 2012, Charles Kane, of the University of Pennsylvania, and co-workers predicted that three-dimensional (3D)

\section{Table I. Quasiparticles in quantum materials.}

Cooper pair Two electrons may become coupled via vibrations of the underlying atomic lattice in conventional (Bardeen-Cooper-Schrieffer) superconductors, producing a composite electron-pair quasiparticle, a boson with integer spin (0 or 1). As bosons, these quasiparticles can condense into a ground state described by a single wave function, producing the phenomenon of superconductivity.

Relativistic Dirac fermion All known fermionic (spin-1/2) particles, such as electrons, are Dirac fermions, described (with their corresponding antiparticles) by Paul Dirac's relativistic quantum wave equation. Quasiparticle relativistic Dirac fermions, such as many-electron excitations in Dirac semimetals, behave as though they have no mass and have the same speed at all energies.

Weyl fermion These are massless fermions related to Dirac fermions. They have a chirality or handedness depending on whether their momentum is aligned or antialigned with their spin. Each Dirac fermion can be resolved into two Weyl fermions of opposite chirality. Never observed as fundamental particles, they are seen as electron quasiparticle excitations in Weyl semimetals.

Laughlin quasiparticle The fractional quantum Hall effect (FQHE) in a 2D "electron gas" involves collective electron quasiparticles that behave as though they have a fractional charge, such as $1 / 3,2 / 5$, or $3 / 7$, the charge on a single electron. Physicist Robert Laughlin proposed this quasiparticle picture of the FQHE in 1983.

Majorana fermion Hypothetical particle proposed in 1937, which is its own antiparticle. These might arise as quasiparticle excitations in superconductors. They are potential ingredients for topological quantum computers.

Anyon Hypothetical particle proposed by Frank Wilczek in 1982, which can have quantum statistics anywhere on a continuum between fermions (half-integer spin) and bosons (integer spin). They might appear as quasiparticles in quantum spin liquids, but have not been definitively identified yet.

Skyrmion Originally predicted as an exotic kind of baryon (the constituents of most ordinary matter) in 1962, skyrmions have been found to exist in the form of vortex-like topological quasiparticle excitations of spins in some magnetic materials.

Dirac semimetals ought to exist: their calculations suggested that a form of bismuth oxide denoted $\beta-\mathrm{BiO}_{2}$ might display this property. ${ }^{3}$ The peculiar electronic properties required for this sort of behavior can be predicted in principle from quantum calculations of band structure, and in the same year, Xi Dai of the Beijing National Laboratory for Condensed Matter Physics in China and his co-workers ${ }^{4}$ showed that these conditions might be satisfied in sodium bismuthate $\left(\mathrm{Na}_{3} \mathrm{Bi}\right)$.

In a Dirac semimetal, there is no energy gap between the conduction and valence bands, but strictly speaking no overlap either: they touch at a single point, where the apices of the "Dirac cones" meet. In $\mathrm{Na}_{3} \mathrm{Bi}$, these electron states are comprised from the atomic electron orbitals of sodium (the $3 s$ orbitals) and bismuth (the $6 p$ orbitals). Classically, we would expect these orbitals to "mix" into hybrid electronic states, which would change the band structure and open up an energy gap: they would become semiconductors (for a small gap) or insulators. But that doesn't happen because certain symmetry properties of the electron states forbid such mixing. As physicist Nai Phuan Ong of Princeton University says, "the electronic properties arise from states that are protected by symmetry." In particular, the conducting states have time-reversal symmetry: it makes no difference if one writes $t$ or $-t$ in their quantum wave functions. It's a little like the way the symmetry of permitted moves prevents bishops in chess 
from crossing onto squares of a different color: it can't be done.

While the Dirac cones in 2D graphene are symmetry-protected, there's an extra degree of such protection in 3D Dirac semimetals such as $\mathrm{Na}_{3} \mathrm{Bi}$, which comes from the topology of the electron states - more akin to the prohibition of transforming a left-handed glove into a right-handed one. Because of this, 3D Dirac semimetals are said to be "topological semimetals."

In 2014, Dai collaborated with Yulin Chen of the University of Oxford in the UK to verify his prediction for $\mathrm{Na}_{3} \mathrm{Bi}^{5}$ Measurements using electron photoemission spectroscopy revealed that the energy and momentum of electrons in the material have the characteristics expected for a 3D Dirac semimetal. Independently and at the same time, two international groups that both included researchers from Princeton University described another 3D Dirac semimetal, cadmium arsenide $\left(\mathrm{Cd}_{3} \mathrm{As}_{2}\right){ }^{6,7}$

Dirac fermions can be regarded as the combination of two related massless particles called Weyl fermions, which have a chiral twist or handedness to them: in a Dirac fermion, the two twists cancel out. To resolve Dirac fermions into their constituent Weyl fermions, one can split them with an applied magnetic field. In effect, the field destroys the time-reversal symmetry, because-for electron-based quasiparticles - it aligns most of the electron spins to break the symmetry: reverse time, and they would have opposite spin. In momentum space, this process corresponds to the splitting of the Dirac cone into two Weyl cones. Formally, this is analogous to the splitting apart of the poles of a magnetic field: the nodes of each Weyl cone then correspond to a "monopole," a hypothetical magnetic entity with just an isolated north or south pole. It's not known how one might create a genuine magnetic monopole - chop a bar magnet in half and you create two new poles at the new ends. But magnetic monopoles are predicted to exist in some fundamental theories in particle physics, such as string theory.

In a quantum material that behaves as a Dirac semimetal, however, you can make the equivalent of these monopoles, namely the corresponding Weyl-fermion quasiparticles. "Weyl nodes must always come in pairs," Ong explains. "Just like magnetic monopoles, it is difficult to pull the pair apart. But an advantage in condensed matter is that one can go to the surface of a crystal where such operations-forbidden in the bulk-may become feasible." In that way, one might create a Weyl semimetal. While such materials would contain Weyl nodes in the bulk, only at the surface is symmetry broken in a way that produces the gapless "kissing" of Weyl cones. In 2015, Zahid Hasan at Princeton and his collaborators predicted that tantalum arsenide (TaAs) might act as a Weyl semimetal, ${ }^{8}$ and shortly thereafter, they verified experimentally, again using photoelectron spectroscopy, that this is so. ${ }^{9}$

One of the reasons for a fundamental interest in these systems is that, as analogues of the particle-physics context in which they were first observed, they should exhibit some of the peculiarities of such systems. Massless Weyl fermions were invoked in the 1960s to explain why particles called neutral pions decay so fast. This decay can create an apparent nonconservation of charge in the chiral fermions, called the chiral anomaly. It was predicted in 1983 that this anomaly might be found in material analogue systems, and in 2015, Ong and his Princeton collaborators saw it in $\mathrm{Na}_{3} \mathrm{Bi}^{10}$

\section{Making new particles in materials}

The 3D Dirac semimetals predicted by Kane in 2012 had another curious feature. They are electrical insulators, but as Ong says, at surfaces different constraints may apply. In this case, the surface electrons may be confined to 2D conducting states: they are metallic. What's more, in these states electron scattering becomes negligible, purely because of the topological shape of the band structure. For this reason, the states are topologically protected: even if there is a considerable amount of disorder or impurities at the material surface, which might be expected to disrupt electrical conductivity in an ordinary material, the conducting states persist. Such materials are said to be topological insulators, and they supply another key focus of current interest in quantum materials. ${ }^{11}$ After the discovery of the first topological insulator, bismuth antimonide $\left(\mathrm{Bi}_{x} \mathrm{Sb}_{1-x}\right),{ }^{12}$ a variety of other candidates have been identified, including bismuth and antimony selenides and tellurides. They are predicted to occur, for example, in a wide range of complex materials called Heusler compounds. ${ }^{13}$

Topological insulators represent a confluence of several themes concerning the exotic electron behavior in materials with unusual topological features of their band structure. "The field of topological insulators is an offspring of QHE physics and quantum spin physics, with hightemperature superconductivity sneaking in some genes," Ong says. But research on topological matter has now expanded well beyond QHE physics, he adds.

For one thing, it has reignited interest in a host of unusual hypothetical particles (see Table I). Take skyrmions, a breed of particle first predicted in the 1960s in a particle-physics context. Skyrmions can be realized as quasiparticles in certain magnetic materials, where they take the form of vortex-like quasiparticle excitations created by topological constraints on the orientation of the magnetic spins. They have been identified in the chiral magnet manganese silicide. ${ }^{14}$

Another exotic particle potentially realizable as a quasiparticle in quantum materials is the anyon. Originally hypothesized by theoretical physicist Frank Wilczek in 1982, the anyon is a strange intermediate between the two conventional classes (like photons) and fermions (like electrons). These are usually regarded as two mutually exclusive classes (although the theory called supersymmetry, for which particle physicists have yet to find validating experimental support, suggests that they can be unified by invoking a particular kind of symmetry). Anyons, however, reject that dichotomy; they can exhibit quantum statistics anywhere between those of bosons and fermions.

Anyon statistics are again a consequence of topological considerations. In effect, exchanging any two such 
particles - the operation that distinguishes the exclusivity of fermions from the sociability of bosons - involves a tangling or "braiding" of their world-lines in spacetime, which means the particles can acquire any arbitrary increment in the phase of their quantum wave functions. For bosons and fermions, in contrast, this phase increment is either 0 or $\pi$, so the two particles either superimpose or cancel exactly. This odd property of anyons, seemingly forbidden in ordinary particle physics, becomes possible for particles confined in two dimensions, which suggests that $2 \mathrm{D}$ quantum materials might be the place to look for the quasiparticle analogues of such objects.

In fact, the vortex-like, fractionally charged quasiparticles observed in the FQHE, seen in thin films of metallic material in the presence of a strong magnetic field, have anyon-like features, as first reported in a fractional quantum Hall fluid in $2005,{ }^{15}$ by observing quantum interference behavior between the quasiparticle states. Good candidates for hosting anyon quasiparticles more directly, however, are the class of quantum materials called quantum spin liquids (see p. 703). They haven't been seen yet, but the hunt is on. ${ }^{16}$ Aside from the fundamental interest, there is good practical reason for wanting to make them, since a certain type of anyon has been proposed as the quantum bit for making error-proof quantum computers called topological quantum computers. ${ }^{17}$

Similar to anyons, Majorana fermions are unusual hypothetical particles that acquire their properties from topological constraints on their "worldlines"- the hypothetical trajectories they can be considered to trace out through spacetime. Again first identified as a theoretical possibility in particle physics, Majorana fermions have the unusual property that, unlike familiar fermions such as electrons, they do not have an antiparticle counterpart because they are their own antiparticle. They too emerge as a prediction of Dirac's relativistic quantum theory.

Like anyons, Majorana fermions could potentially serve as qubits for topological quantum computers, in which they would be controlled and manipulated to process information. However, making them is still a challenge. Whereas anyons are predicted in 2D systems, Majorana fermions are zero-dimensional. In one view, they are predicted to appear at the ends of a one-dimensional conducting nanowire coupled to a superconducting electrode, in the presence of a magnetic field. ${ }^{18}$ Possible signatures of such quasiparticles have been reported for indium antimonide (InSb) and arsenide (InAs) nanowires with superconducting contacts, although the evidence still falls short of definitive proof. ${ }^{19,20}$

The manifestation of such exotic behaviors induced by topological constraints on the electronic degrees of freedom constitutes "the biggest intellectual leap" that quantum materials represent, according to physicist Stephen Blundell of the University of Oxford. It shows that "what seemed to be a peculiarity of the quantum Hall effect can be realized in many materials in less stringently controlled experimental systems," he says.

"Topologically protected states have the advantage that they are resistant to disorder and defects and therefore can persist in the sometimes messy condition that afflicts real materials," Blundell adds. "It's not yet clear what they might be useful for, but topological quantum computing is a possibility." It was recently shown that, at least in one dimension, symmetry-protected topological phases of matter quite generally contain quantum "resources" that can be exploited for efficient computation; ${ }^{21}$ they can be considered a "computational phase of matter."

\section{Quantum criticality}

One of the most celebrated features of quantum theory is uncertainty: crudely, the idea that you cannot simultaneously know everything that is knowable in principle about a quantum system. This notion, enshrined in Werner Heisenberg's uncertainty principle, is not an expression of ignorance or clumsiness - it's not that we can't measure properties carefully enoughbut rather reflects the fact that some properties of quantum systems can't simultaneously exist in a well-defined way alongside others.
A consequence of quantum uncertainty is that some properties of a quantum system may fluctuate even at zero temperature. Such behavior is predicted for a wide range of systems that demand a quantum description, including superconductors and heavy-fermion metals - intermetallic compounds with $f$ orbital electrons, such as $\mathrm{CeCu}_{6}, \mathrm{CeAl}_{3}$, and $\mathrm{UPt}_{3}$, in which the charge carriers are quasiparticles of strongly correlated electrons with a collective mass much greater than that of individual electrons. Such systems have two possible states even at absolute zero: an orderly one (such as a magnetically ordered material), and one in which quantum fluctuations disrupt the order. The two states are separated by a quantum phase transition demarcating the point at which fluctuations dominate. This transition is entirely analogous to the critical points known in classical statistical mechanics, for example in ferromagnets and liquids and gases, where the critical point marks the end of any clear distinction between the two phases. The difference is that in quantum criticality, the fluctuations are not thermal but driven by quantum uncertainty.

Quantum criticality has emerged as a rather general framework for understanding quantum behavior in diverse materials. ${ }^{22}$ It has been seen, for example, in the magnetic states of heavy-fermion systems such as $\mathrm{CeCu}_{6} \cdot{ }^{23}$ The surprising thing is that a quantum critical point does not go away at temperatures above zero; the region in which quantum fluctuations overwhelm the system is predicted to broaden at finite temperatures.

"It is this influence that elevates quantum criticality from an intellectual abstraction at absolute zero to a real-world phenomenon that can profoundly change finite-temperature material properties," according to physicists Piers Coleman and Andrew Schofield. ${ }^{24}$

Just as cosmic black holes distort the surrounding spacetime, quantum critical points distort the "fabric" of the phase diagram, creating a V-shaped region of "quantum critical matter" fanning out at finite temperatures from the quantum critical point. Such a broadening regime of quantum criticality was reported in 
2014 in the magnetic material $\mathrm{CoNb}_{2} \mathrm{O}_{6}$, in which the magnetic spins are coupled in one-dimensional chains. ${ }^{25}$

The fluctuations of a quantum critical point can drive electrons to reorganize themselves close to the transition into new phases. For this reason, Coleman and Schofield say, "quantum criticality may be a highly effective catalyst for the formation of new stable types of material behavior, providing an important new route for the design and discovery of new classes of material."

One particularly important class of materials thought to feel the influence of quantum criticality is high-temperature superconductors. ${ }^{26}$ Nicholas Butch of the University of Maryland and coworkers have reported a signature of quantum criticality in the copper-oxide ceramic $\mathrm{La}_{2-\mathrm{x}} \mathrm{Ce}_{\mathrm{x}} \mathrm{CuO}_{4},{ }^{27}$ with the superconducting phase separated from a conventional "Fermi liquid" metallic phase by a region of "non-Fermi liquid" behavior at temperatures below the normal superconducting transition temperature of around $10-17 \mathrm{~K}$, where there are strong fluctuations of the copper spins. The researchers concluded that quantum criticality plays a significant role in shaping the phase diagram of this and other electron-doped copper-oxide systems. There are two quantum critical points in this system, closely spaced in the phase space defined by the temperature, doping level, and applied magnetic field. Their effects compete: one stabilizes the superconducting phase, the other originates from the suppression of superconductivity by a magnetic field, and between them, they define the region of phase space where superconductivity pertains. It's also thought that quantum criticality plays a role in the "unconventional" superconductivity of some other systems with strongly correlated electrons, such as iron pnictides ${ }^{28}$ and heavy-fermion metals. $^{29}$

The latter materials are one of the beststudied systems in which strong correlations between electrons create quasiparticle behavior, with the effective charge carriers having many times the mass of an electron. Many have rich phase diagrams at low temperatures where quantum

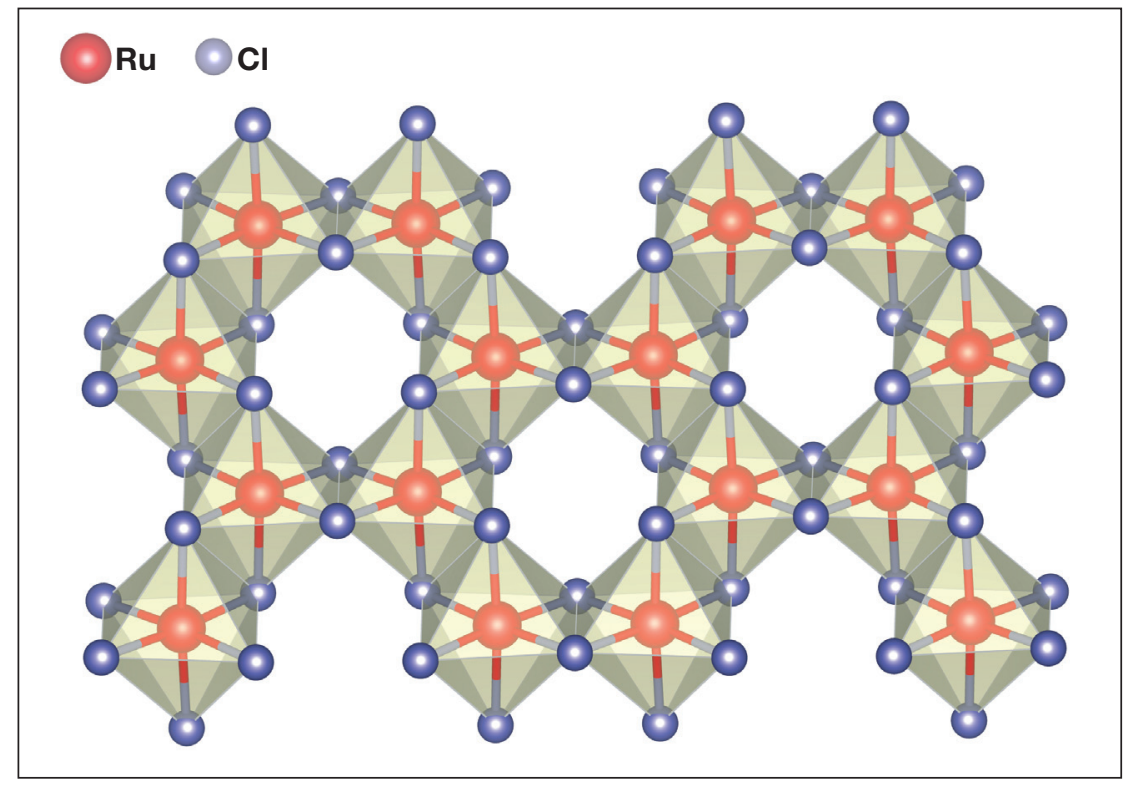

Figure 2. Honeycomb crystal structure of the candidate quantum spin liquid $\alpha-\mathrm{RuCl}_{3}$. Image courtesy of Arnab Banerjee, Oak Ridge National Laboratory.

effects dominate. In addition to superconductivity and quantum critical points, some heavy-fermion metals exhibit the so-called Kondo effect: an interaction between a local magnetic moment created by $f$-shell electrons and the spins of the delocalized conduction electrons in the material. This interaction can lead to the opening up of a small bandgap, making the material insulating - a Kondo insulator - at very low temperatures. The first Kondo insulator to be recognized, samarium hexaboride $\left(\mathrm{SmB}_{6}\right)$, was discovered almost 50 years ago; ${ }^{30}$ many others have subsequently been found.

In a further illustration of the deep connections between the cluster of ideas that underpin quantum materials, it was recognized in 2010 that Kondo insulators may possess topologically protected metallic surface states: they are versions of topological insulators. ${ }^{31,32}$ This behavior was confirmed experimentally in $\mathrm{SmB}_{6}$ two years later. ${ }^{33,34}$ "Topological Kondo insulators tie together the nominally disparate subfields of strong electron correlations and topological electronic states," Butch says. These exotic materials might never find "applications" in the technological sense; their value is rather to show how various parts of the story of quantum materials fit together.

\section{Spin liquids and quantum magnetism}

One might say the same about another class of quantum materials, called quantum spin liquids. In the 1970s, Nobel laureate physicist Philip W. Anderson pointed out that some magnetic materials might be geometrically incapable of aligning all their spins in a single, most stable manner to form a well-defined, orderly magnetic phase. Imagine an antiferromagnet—in which adjacent spins prefer to be oppositely oriented - on a triangular lattice. Each spin has two nearest neighbors in a triangle, but the antiparallel alignment cannot be satisfied for all of the trio. One possibility is that the spin lattice freezes into a disordered "glassy" state, but Anderson showed that quantum mechanics allows the possibility of fluctuating spins even at absolute zero. This state is called a quantum spin liquid, and Anderson later suggested that it might be connected to high-temperature superconductivity in copper oxides. ${ }^{35}$

"A spin glass is a structure where spins freeze in an effectively disordered configuration at some non-zero temperature," explains Stephen Nagler of Oak Ridge National Laboratory (ORNL) in Tennessee. "Spin liquids, on the other hand, remain disordered down to zero 
temperature but do not freeze as such at a non-zero temperature." In quantum spin liquids, this dynamical disorder is quantum mechanical in origin: the spins exhibit zero-point motion, lacking a stationary ground state even at absolute zero.

"Quantum spin liquids are currently where some of the most active theoretical and experimental work [on quantum materials] is going on," Blundell says. However, they are hard to study experimentally. "The problem with realizing spin liquids experimentally is that they are balanced on a knife edge," Blundell explains. "When they are cooled down, they should show no order even down to absolute zero, but as you cool them down, all sorts of minor effects that you can ignore at high temperature start to come into play."

Quantum spin liquids should appear in materials with particular kinds of magnetic structures (e.g., antiferromagnets with triangular lattices). Nagler and his co-workers at ORNL have reported the possible signature of one such variety of a quantum spin liquid, revealed by neutron scattering from both powder ${ }^{36}$ and single crystals ${ }^{37}$ in the alpha phase of ruthenium trichloride $\left(\alpha-\mathrm{RuCl}_{3}\right)$ (see Figure 2), which has a layered quasi-2D hexagonal structure. This is not itself a spin liquid, but it is thought to be close to such a state-specifically, close to a special theoretical case called a Kitaev spin liquid, in which the spins exist on a 2D honeycomb lattice that is frustrated, meaning that there's no way of aligning the spins so that each enjoys the most favorable interactions with all its neighbors. The Kitaev spin liquid model was once thought to be rather artificial, but it's now thought that not just $\mathrm{RuCl}_{3}$ but also some iridate compounds might be good candidates for embodying it. ${ }^{38}$ What's more, there is evidence that quantum spin liquids exist in other geometrically frustrated triangular-lattice materials, including the natural copper mineral herbertsmithite, $\mathrm{Ca}_{10} \mathrm{Cr}_{7} \mathrm{O}_{28}{ }^{39}$ and $\mathrm{YbMgGaO}_{4}{ }^{40}$

But the signature of a true quantum spin liquid is not very clear. When a material enters this state, there is no change in the symmetry of the system, and so one does not see the marked signatures typical of other magnetic phase transitions, such as spikes in the thermodynamic quantities like specific heat. And the excitations of a quantum spin liquid at low temperature are not well-defined in the same way as in ordinary magnets, Nagler says. As a result, he admits, "I think it is still somewhat of an open question as to whether true quantum spin liquids have been observed experimentally." What's more, a quantum spin glass is strictly defined only at absolute zero, but no one can make measurements there.

The magnetic and electronic structures can become coupled in interesting ways in such materials. Quantum spin liquids may have Dirac nodes like those of Dirac semimetals, and the electrons are expected to congregate into quasiparticles with fractional charge, like those in the FQHE. "Mostly, quantum spin liquids are intellectually interesting," as opposed to having obvious practical value, says physicist Steven Kivelson of Stanford University. "They are entirely new phases of quantum matter, generalizing notions developed in the context of the FQHE and conventional superconductors - both of which are states with considerable structure in common with spin liquids."

Still, applications are not entirely out of the picture. For example, the Kitaev model for quantum spin liquids predicts that the electrons could form excited-state, topologically protected quasiparticles corresponding to Majorana fermions, raising the possibility of using quantum spin liquids for topological quantum computing. But that's a dim prospect at best. "Perhaps it is possible that this type of research will one day lead to a useful technology for quantum computing or other applications," Nagler says, "but we are a long way off from using quantum spin liquids in this way." However, he adds, "we are breaking ground in elucidating the behavior of complex materials, and in the long run, I believe that knowledge is very likely to be useful in some fashion, perhaps one that we have not yet thought of."

\section{Emergence}

Today one can hardly pick up a journal that publishes condensed-matter physics without seeing a rash of papers on quantum materials, particularly those whose electronic properties are influenced by topological factors. Yet if anything, the field seems ready to expand further. Andrei Bernevig of Princeton University and co-workers recently revealed how much space there is to expand into, and identified a wide range of new candidate topological and strongly correlated materials by combining chemical intuition with physical theory to find a shortcut to the prediction of interesting band structures ${ }^{41}$ —in effect, a tractable theory of topological quantum chemistry. They identified several known materials likely to yield such phases, such as strained lead suboxide $\mathrm{Pb}_{2} \mathrm{O}$. The electron correlations that are essential to the behavior of many of these quantum materials mean that one cannot understand them by thinking about how electrons behave in isolation. The electronic behaviors are an emergent property, much as is the flocking of birds or the mound building of termites. The collective phenomena cannot be deduced simply by adding up the behaviors of individual constituents. In lieu of "quantum materials," says Butch, "personally I prefer the term emergence, and I'm not sure why it didn't catch on." The concept of emergent or collective behavior, he says, "was a foundation of strongly correlated electron research" - in superconductors and heavy fermions, for example - " "and it has been recognized as an important concept in topological materials."

Perhaps this is the way to delimit the otherwise almost indefinite scope of quantum materials. Some researchers would like to see the term used very broadly to cover all materials whose properties are strongly dictated by quantum mechanics - for example, quantum dots, particles of matter so small that the energy states of the electrons are altered from their bulk values by the quantum mechanical influence of confinement. But those effects can be understood by considering the states of single electrons, in contrast to the quasiparticles that typify most quantum materials.

Are such quantum-confinement effects then to be considered one of the "exotic physical properties" included in the DOE definition? The loose wording might make that a matter of personal preference. But this looseness is what recommends the definition to some researchers. "It's about 
right," Blundell says. "Of course, in a sense, all materials are quantum, but I think what we have in mind when we talk about a quantum material is those cases when specific material properties derive more spectacularly from seemingly unusual quantum states."

Others are less sanguine. "I'm not sure the DOE definition is that helpful," Ceperley says. "'Exotic' is a term that depends on your reference. It reminds me of similar discussions over the term "nano."' Ceperley thinks that another definition would be "materials whose properties are not well described by independent electron theory," echoing Butch's focus on collectivity and emergence. But Kivelson, who is a founding editor of the new journal $n p j$ Quantum Materials, takes a wider view, seeing the field as "a very broad set of topics having largely to do with the electronic properties of novel solids and solid-state devices in which the quantum character of the electrons is important." It is, Kivelson suggests, really not much more or less than "sort of what used to be called 'hard condensed-matter physics."”

What's in a name, though? The bigger issue is the way research on quantum materials is forging new links between diverse disciplines and ideas, expanding the materials universe while at the same time showing how common themes govern the properties of matter, so that substances with compositions and structures that might make the theoretical physicist blanch are, after all, more familiar than they might seem. As the 19th century essayist Ralph Waldo Emerson put it, "Nature is an endless combination and repetition of a very few laws. She hums the old well-known air through innumerable variations."

\section{References}

1. K.S. Novoselov, A.K. Geim, S.V. Morozov, D. Jiang, M.I. Katsnelson, I.V. Grigorieva, S.V. Dubonos, A.A. Firsov, Nature 438, 197 (2005).

2. Y. Wang, D. Wong, A.V. Shytov, V.W. Brar, S. Choi,
Q. Wu, H.Z. Tsai, W. Regan, A. Zettl, R.K. Kawakami, S.G. Louie, L.S. Levitov, M.F. Crommie, Science $\mathbf{3 4 0}$, 734 (2013).

3. S.M. Young, S. Zaheer, J.C.Y. Teo, C.L. Kane, E.J. Mele, A.M. Rappe, Phys. Rev. Lett. 108, 140405 (2012).

4. Z. Wang, Y. Sun, X.-Q. Chen, C. Franchini, G. Xu, H. Weng, X. Dai, Z. Fang, Phys. Rev. B 85, 195320 (2012).

5. Z.K. Liu, B. Zhou, Y. Zhang, Z.J. Wang, H.M. Weng, D. Prabhakaran, S.K. Mo, Z.X. Shen, Z. Fang, X. Dai, Z. Hussain, Y.L. Chen, Science 343, 864 (2014).

6. M. Neupane, S.-Y. Xu, R. Sankar, N. Alidoust, G. Bian, C. Liu, I. Belopolski, T.-R. Chang, H.-T. Jeng, H. Lin, A. Bansil, F. Chou, M.Z. Hasan, Nat. Commun. 5, 3786 (2014)

7. S. Borisenko, Q. Gibson, D. Evtushinsky, V. Zabolotnyy, B. Buechner, R.J. Cava, Phys. Rev. Lett. 113, 027603 (2014).

8. S.-M. Huang, S.-Y. Xu, I. Belopolski, C.-C. Lee, G. Chang, B. Wang, N. Alidoust, G. Bian, M. Neupane, C. Zhang, S. Jia, A. Bansil, H. Lin, M.Z. Hasan, Nat. Commun. 6, 7373 (2015)

9. S.-Y. Xu, I. Belopolski, N. Alidoust, M. Neupane, G. Bian, C. Zhang, R. Sankar, G. Chang, Z. Yuan, C.-C. Lee, S.-M. Huang, H. Zheng, J. Ma, D.S. Sanchez, B. Wang, A. Bansil, F. Chou, P.P. Shibayev, H. Lin, S. Jia, M.Z. Hasan, Science 349, 613 (2015).

10. J. Xiong, S.K. Kushwaha, T. Liang, J.W. Krizan, M. Hirschberger, W. Wang, R.J. Cava, N.P. Ong, Science 350, 413 (2015).

11. M.Z. Hasan, C. L. Kane, Rev. Mod. Phys. 82, 3045 (2010).

12. D. Hsieh, D. Qian, L. Wray, Y. Xia, Y.S. Hor, R.J. Cava, M.Z. Hasan, Nature 452, 970 (2008).

13. S. Chadov, X. Qi, J. Kübler, G.H. Fecher, C. Felser, S.C. Zhang, Nat. Mater. 9, 541 (2010).

14. S. Mühlbauer, B. Binz, F. Jonietz, C. Pfleiderer, A. Rosch, A. Neubauer, R. Georgil, P. Böni, Science 323, 915 (2009).

15. F.E. Camino, W. Zhou, V.J. Goldman, Phys. Rev. B 72, 075342 (2005).

16. S.C. Morampudi, A.M. Turner, F. Pollmann, F. Wilczek, Phys. Rev. Lett. 118, 227201 (2017).

17. L. Venema, Nature 452, 803 (2008).

18. Y. Oreg, G. Refael, F.V. Oppen, Phys. Rev. Lett. 105, 177002 (2010).

19. V. Mourik, K. Zuo, S.M. Frolov, S.R. Plissard, E.P. Bakkers, L.P. Kouwenhoven, Science 336, 1003 (2012).

20. A. Das, Y. Ronen, Y. Most, Y. Oreg, M. Heiblum, H. Shtrikman, Nat. Phys. 8, 887 (2012).

21. D.T. Stephen, D.-S. Wang, A. Prakash, T.-C. Wei, R. Raussendorf, Phys. Rev. Lett. 119, 010504 (2017).
22. S. Sachdev, B. Keimer, Phys. Today 64, 29 (2011).

23. H. von Löhneysen, T. Pietrus, G. Portisch, H.G. Schlager, A. Schröder, M. Sieck, T. Trappmann, Phys. Rev. Lett. 72, 3262 (1994).

24. P. Coleman, A.J. Schofield, Nature 433, 226 (2005).

25. A.W. Kinross, M. Fu, T.J. Munsie, H.A. Dabkowska, G.M. Luke, S. Sachdev, T. Imai, Phys. Rev. X 4, 031008 (2014).

26. S. Sachdev, Phys. Status Solidi B247, 537 (2010).

27. N.P. Butch, K. Jin, K. Kirshenbaum, R.L. Greene, J. Paglione, Proc. Natl. Acad. Sci. U.S.A. 109, 8440 (2012).

28. P. Walmsley, C. Putzke, L. Malone, I. Guillamón, D. Vignolles, C. Proust, S. Badoux, A.I. Coldea, M.D. Watson, S. Kasahara, Y. Mizukami, T. Shibauchi, Y. Matsuda, A. Carrington, Phys. Rev. Lett. 110, 257002 (2013).

29. P. Gegenwart, Q. Si, F. Steglich, Nat. Phys. 4, 186 (2008).

30. A. Menth, E. Buehler, T.H. Geballe, Phys. Rev. Lett. 22, 295 (1969).

31. M. Dzero, K. Sun, V. Galitski, P. Coleman, Phys. Rev. Lett. 104, 106408 (2010).

32. M. Dzero, J. Xia, V. Galitski, P. Coleman, Annu. Rev. Condens. Matter Phys. 7, 249 (2016).

33. S. Wolgast, C. Kurdak, K. Sun, J.W. Allen, D.J. Kim, Z. Fisk, Phys. Rev. B 88, 180405 (2013).

34. J.D. Thomas, T. Grant, J. Botimer, Z. Fisk, J. Xia, Sci. Rep. 3, 3150 (2014).

35. P.W. Anderson, Science 235, 1196 (1987).

36. A. Banerjee, C.A. Bridges, J.-Q. Yan, A.A. Aczel, L. Li, M.B. Stone, G.E. Granroth, M.D. Lumsden, Y. Yiu, J. Knolle, S. Bhattachaarjee, D.L. Kovrizhin, R. Moessner, D.A. Tennant, D.G. Mandrus, S.E. Nagler, Nat. Mater. 15, 733 (2016).

37. A. Banerjee, J. Yan, J. Knolle, C.A. Bridges, M.B. Stone, M.D. Lumsden, D.G. Mandrus, D.A. Tennant, R. Moessner, S.E. Nagler, Science $\mathbf{3 5 6}$, 1055 (2017).

38. M. Hermanns, I. Kimchi, J. Knolle, Cornell University Library (2017), www.arxiv.org/abs/1705.01740.

39. C. Balz, B. Lake, J. Reuther, H. Luetkens, R. Schönemann, T. Herrmannsdörfer, Y. Singh, A.T.M. Nazmul Islam, E.M. Wheeler, J.A. Rodriguez-Rivera, T. Guidi, G.G. Simeoni, C. Baines, H. Ryll, Nat. Phys. 12, 942 (2016).

40. J.A.M. Paddison, M. Daum, Z. Dun, G. Ehlers, Y. Liu, M.B. Stone, H. Zhou, M. Mourigal, Nat. Phys. 13, 117 (2017).

41. B. Bradlyn, L. Elcoro, J. Cano, M.G. Vergniory, Z. Wang, C. Felser, M.I. Aroyo, B.A. Bernevig, Nature 547, 298 (2017).

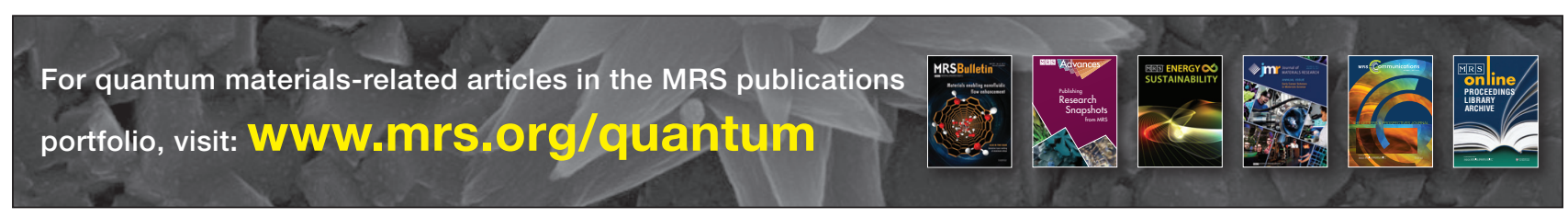

